\title{
Eficacia de una intervención cognitivo-conductual de inoculación al estrés para la disminución de TEPT en mujeres víctimas de abuso sexual en Hidalgo
}

\author{
Effectiveness of a cognitive-behavioural inoculation stress treatment for the \\ reduction of PTSD in women who have suffer sexual assault in Hidalgo State \\ Wendy Guadalipe Soto-Zentella ${ }^{a}$, Dalia Irazu Zarco-Cárdenas ${ }^{b}$, Sandra Mayela López- \\ Martínez $^{c}$, Gelacio Guzmán-Díaz ${ }^{d}$, Jesús Cisneros-Herrera ${ }^{e}$
}

\begin{abstract}
:
Sexual violence is a problem that affects mainly women, and it is related to posttraumatic stress disorder, which is made of acute anxiety, dissociative reactions, situation avoidance and cognitive alterations, amongst other symptoms, that affect severely the life of a person. Stress inoculation is a cognitive-behavioural treatment the uses psicoeducation, relaxation techniques, cognitive restructuring and social skills training. In this research 10 women, elected for convenience, who have suffered a sexual assault, participated. Results show that symptoms of re-experiencing, avoidance, cognitive alterations, physiological reactivity, dissociation and dysfunctionality in daily life decreased. The conclusion is that this cognitive-behavioural treatment is effective to treat symptoms of posttraumatic stress disorder.
\end{abstract}

Keywords:

Cognitive-behavioural treatment, posttraumatic stress disorder, stress inoculation,

Resumen:

La violencia sexual es un problema que afecta principalmente a mujeres y se relaciona con el trastorno de estrés postraumático, que se caracteriza por una intensa angustia, reacciones disociativas, evitación de situaciones y alteraciones cognitivas, entre otros síntomas, que afectan gravemente la vida de la persona. La inoculación del estrés es un tratamiento cognitivo-conductual que emplea a la psicoeducación, técnicas de relajación, la restructuración cognitiva y el entrenamiento en habilidades sociales. En esta investigación participaron 10 mujeres elegidas por conveniencia que habían sufrido de abuso sexual. Los resultados indican que los síntomas de reexperimentación, evitación, alteraciones cognitivas, reactividad fisiológica, disociación y disfuncionalidad en la vida cotidiana disminuyeron. Se concluye que este tratamiento cognitivo-conductual es eficaz para tratar los síntomas del trastornos de estrés postraumático.

\section{Palabras Clave:}

Inoculación del estrés, trastorno de estrés postraumático, tratamiento cognitivo-conductual

\section{Introducción}

La violencia sexual es un problema que trasciende y a afecta todos los sectores de la sociedad, principalmente al género femenino en aspectos físicos, psicológicos y sociales. Estos actos han conllevado a que las mujeres con el paso del tiempo presenten síntomas del trastorno por estrés postraumático, referido del DSM-V el trastorno por estrés postraumático, es la exposición a un evento

\footnotetext{
Autor de Correspondencia, Universidad Autónoma del Estado de Hidalgo, https://orcid.org/0000-0003-2166-4239, Email: so316729@uaeh.edu.mx

b Universidad Autónoma del Estado de Hidalgo, https://orcid.org/0000-0002-4197-0383, Email: za383592@uaeh.edu.mx c Universidad Autónoma del Estado de Hidalgo, https://orcid.org/0000-0003-0697-9208, Email: lo383446@uaeh.edu.mx d Universidad Autónoma del Estado de Hidalgo, https://orcid.org/0000-0003-4869-8658, Email: gelacio_guzman@uaeh.edu.mx e Universidad Autónoma del Estado de Hidalgo, https://orcid.org/0000-0003-1370-8086, Email: jesus_cisneros@uaeh.edu.mx
} 
traumático como lo es la violencia sexual en una o más afecciones de manera directa, la investigación se enfocó en analizar los síntomas del trastorno de estrés postraumático mediante la prueba que evalúa los niveles de TETP denominada EGS-R antes y después de una intervención cognitivo- conductual basada en la técnica de inoculación al estrés, técnica que combina diferentes elementos de enseñanza, reestructuración cognitiva, ensayos conductuales e imaginarios, creada para generar y desarrollar habilidades de afrontamiento de manera inmediata 0 para dificultades futuras (Meinchenbaun,1987), como lo presentan las víctimas de abuso sexual debido a que muchas de ellas no hablaron de sus síntomas por mucho tiempo y en su vida cotidiana actual están presentando problemas en sus diferentes áreas. Guerra y Barrera (2017) mencionan que esta técnica ha sido útil en niños y adolescentes, en esta ocasión fue aplicada a mujeres en un rango de edad de entre 25 a 40 años de edad, se trabajó bajo el método cuantitativo con una muestra de tipo no probabilístico debido a que se eligió en base a los síntomas de toda la población del TETP, el caso del presente estudio manejo las variable dependiente como el estrés postraumático, para investigar y medir, la variable independiente que en este caso se manejó como el modelo de intervención, ya que es, la que genero un cambio en la disminución de los síntomas en las mujeres que presentan trastorno de estrés postraumático.

\section{Trastorno de estrés postraumático}

De acuerdo al DSM-5 (APA, 2014) el Trastorno de Estrés Postraumático (TEPT) es caracterizado por síntomas que han sido resultado de una experiencia directa, observación o conocimiento de un suceso traumático. Estos sucesos ponen en peligro la integridad física y psicológica de las personas a tal grado que son incapaces de afrontar tales situaciones por sí mismas (Echeburúa, 2016).

\section{Repercusiones y síntomas del estrés postraumático}

De acuerdo con el DSM-5 (APA, 2014) los síntomas del estrés postraumático que se presentan en los sujetos son: - Recuerdos angustiosos recurrentes, involuntarios, en intrusivos del suceso (s) traumático (s).

- Sueños angustiosos recurrentes en los que el contenido y/o el efecto del sueño está relacionado con el suceso (s) traumático (s)

- Reacciones disociativas: el sujeto siente o actúa como si se repitiera el suceso traumático

- Malestar psicológico intenso o prolongado al exponerse a factores internos o externos que simbolizan o se parecen a un aspecto de suceso traumático (miedo, terror, enfado, vergüenza)

- Reacciones fisiológicas intensas a factores internos o externos que simbolizan o se parecen a un aspecto de suceso (s) traumático (s).

- Evitación persistente a estímulos asociados al suceso (s) traumático

- $\quad$ Alteraciones negativas cognitivas y del estado del ánimo

- $\quad$ Alteración importante de la alerta y reactividad

Este trastorno tiene efecto en las diferentes esferas de la vida de los individuos, quienes lo presentan tienen menores ingresos, altos índices de falla escolar y problemas interpersonales comparados con la población en general, sin mencionar otros. (Palacios y Heinze 2002).

Siguiendo a Palacios y Heinze (2002) el TEPT tiene consecuencias negativas en sus vidas tales como:

- Sentirse culpable por cosas que hicieron o no hicieron, por sentimientos experimentados 0 no experimentados, pensamientos y/o creencias falsas. En caso de agresión física, por no sentir suficiente ira hacia el agresor o por no haber abandonado antes la relación.

- $\quad$ Pensar que el mundo ya no es un lugar seguro, que no puede confiar en otros, ya que cualquier lugar es peligroso y no se puede fiar de las personas.

- Las personas con trauma complejo muestran un severo déficit en la experimentación de emociones positivas como el amor o la alegría, así como poca capacidad para establecer relaciones interpersonales significativas; éstas se ven disminuidas por la pasividad la persona traumatizada y una oscilación entre la evitación y la revictimización (Herman, 2015).

\section{Prevalencia de estrés postraumático y factores de riesgo}

De acuerdo a Palacios y Heinze (2002) no todo aquel que se expuso a un evento traumático desarrolla el TEPT. Su prevalencia en la población general es de 8 a $9 \%$, y las mujeres son quienes corren un mayor riesgo para desarrollarlo, así como es más frecuente después de ciertos eventos traumáticos como una violación.

Como menciona el DSM 5 (APA, 2014) los factores de riesgo se dividen generalmente en; Factores pretraumaticos (como problemas emocionales en la infancia, bajo nivel socioeconómico, exposición a un trauma previo, etc.), factores peritraumáticos (la gravedad del trauma, el peligro percibido, la violencia interpersonal, etc.) y factores postraumáticos (recuerdos repetitivos relacionados al trauma, estrategias de afrontamiento inadecuadas, etc.). 


\section{Desarrollo y curso del estrés postraumático}

De acuerdo al DSM-V (2014) el trastorno de estrés postraumático puede ocurrir a cualquier edad, empezando a partir del primer año de vida. Los síntomas generalmente comienzan dentro de los primeros 3 meses después del trauma, aunque puede haber un retraso de meses, o incluso de años, antes de que se cumplan los criterios para el diagnóstico. La duración de los síntomas también varía, con una recuperación completa a los 3 meses en aproximadamente la mitad de los adultos, mientras que otras personas permanecen sintomáticas durante más de 12 meses y a veces durante más de 5 años.

\section{Violencia sexual}

La Organización Mundial de la Salud (OMS, 2013) define la violencia sexual como todo acto sexual, el intento de llevar a cabo un acto sexual, las insinuaciones sexuales no deseadas, o utilizar la sexualidad de una persona mediante la coerción por otro individuo, independientemente de la relación que tengan con la víctima. Por otra parte, para Muruaga (2018) la violación es la forma más referida de violencia sexual, en muchas sociedades se define como el contacto sexual con otra persona sin su consentimiento. Es la agresión cometida cuando la resistencia de la víctima es dominada por la fuerza o cualquier otra forma de coerción.

Las estimaciones mundiales publicadas por la OMS (2017) indican que alrededor de una de cada tres mujeres en el mundo (35\%) han sufrido violencia física y/o sexual de pareja o violencia sexual por terceros en algún momento de su vida.

Este tipo de violencia ha incrementado a comparación de otros años. De acuerdo con el Instituto Nacional de Estadística y Geografía (INEGI, 2016) los principales delitos cometidos en contra de las mujeres son los relacionados con el abuso sexual (42.6\%) y la violación (37.8 por ciento). Son 10, 807941 (23.2\%) mujeres que han sufrido violencia sexual, en el año 2016, y 19, 216151 $(42.3 \%)$ las que han sufrido violencia sexual alguna vez en su vida.

Ramos et al., (2001) menciona que la violencia sexual es generada mayormente por personas pertenecientes al ámbito familiar que por personas externas al círculo familiar. Las victimas que han sido entrevistadas en su estudio manifiestan que sus victimarios en muchas ocasiones fue su padre, hermano, tíos o abuelos y en casos extraordinarios suelen ser vecinos o personas de lugares cercanos a su comunidad. Solo alrededor del 5\% de la población de víctimas adultas de violencia sexual reportan el incidente a la policía, sin embargo, el otro 95\% de la población no reporta el abuso sexual por miedo, vergüenza, o por sentirse culpadas por comentarios como "ella lo provoco" (Contreras et al.,2010).

\section{Violencia sexual a la mujer y estrés postraumático}

La violencia sexual en mujeres, ya sea en la edad adulta o en la infancia, marca totalmente la vida de la víctima; tanto a nivel físico, psicológico como social y puede causar una serie de trastornos mentales, como lo es el estrés postraumático (Rozo, 2019).

De acuerdo a Lozano, et al. (2017) está claro que la violencia sexual es un gran estresor y un acontecimiento traumático que puede provocar trastorno de estrés postraumático, entre otras consecuencias psicológicas. En México, Medina, et al. (2005) encontraron que el 68\% de la población ha estado expuesta al menos a un suceso estresante en su vida. Siendo la violación, acoso y abuso sexual más frecuentes en mujeres. El 2.3\% de las mujeres y el $0.49 \%$ de los hombres presentaron un trastorno de estrés postraumático con mayores manifestaciones a causa de violaciones, secuestro y abuso sexual, por lo que recomiendan ampliar los tratamientos para atender las secuelas de la violencia.

Muchas de mujeres que logran sobrevivir tras ser violadas sexualmente, por lo regular, no lo externalizan a nadie y mucho menos reciben atención psicológica debido a que no está en sus posibilidades (Medina, et al., 2005). Es por tal motivo que se decidió trabajar con estrés postraumático, pues frecuentemente dichas mujeres pueden desarrollarlo, lo que impide que puedan continuar funcionalmente con su vida.

\section{Intervención cognitivo conductual}

El modelo cognitivo parte de la premisa de que no es la situación misma la que determina, de manera directa, la forma en que uno siente y se comporta sino es la interpretación que se le da a esa situación. Mientras que el modelo cognitivo conductual no solo se concentra en los procesos del pensamiento, sino también presta atención en las emociones y los procesos motivacionales complejos, de este modo, se fundamenta en que la conducta puede ser causada en función de las cogniciones y la modificación de conducta solo se podrá lograr alterando los procesos cognitivos disfuncionales (Beck, 2000; Gabalda, 2009). El terapeuta será el que se encargará de enseñarle al paciente a reconocer, evaluar y modificar sus pensamientos para así lograr un alivio de los síntomas y que, posteriormente, el paciente por sí mismo sea su propio terapeuta (Beck, 2000).

El modelo cognitivo conductual es un tratamiento con soporte empírico, pues existe una amplia evidencia de su utilidad y efectividad, así como se ha empleado en diversos trastornos (Baguena, 2001; Pujadas y Pérez, 2013; Gonzáles y Javato, 2015; Guerra y Barrera, 2017). En un estudio realizado por Puerta y Padilla (2011) se comprobó la efectividad del tratamiento cognitivo conductual (TCC) en el trastorno de depresión, por lo que 
recomiendan promover esta terapia con otro tipo de trastornos.

El estudio de Padilla (2014) muestra un caso clínico especializado sobre la eficacia del tratamiento cognitivoconductual al trastorno de ansiedad generalizada en la adolescencia, demostrando que este tipo de tratamiento logra disminuir los síntomas de manera rápida y eficaz, sin embargo, recomiendan realizar seguimiento meses después del tratamiento para examinar cuáles son las mejoras que siguen vigentes.

Al igual se han realizado investigaciones sobre la eficacia que tiene para la bulimia nerviosa, concluyendo que este tipo de tratamiento es una de las mejores alternativas para los trastornos de la alimentación, pues ayuda a mantener estilos de vida saludable (Gonzáles y Javato, 2015).

Por otra parte, en otras investigaciones realizas a pacientes con un primer episodio de psicosis, concluyeron que la TCC parece ser beneficiosa para la disminución de síntomas psicóticos y para mejorar la calidad de vida de estos pacientes (Jackson, et al., 2008; Travé y Pousa, 2012).

Con relación específicamente al estrés postraumático en mujeres víctimas de una violación, se realizó un estudio por Cáceres, et al., (2011) a 73 mujeres, en dónde evaluaron la eficacia de dicho tratamiento a través del estadístico $\mathrm{g}$ de Hedges que señala el efecto de cada variable dependiente (en ese caso los síntomas del estrés postraumático). Los resultados reflejaron que mayoría de las mujeres disminuyeron su sintomatología de manera rápida a través de este tipo de tratamiento, pues al principio contaban con todo el cuadro clínico de TEPT y tras realizar la intervención lograron disminuir los síntomas, siendo únicamente 3 mujeres las que continuaron con TETP.

Por su parte, Guerra y Plaza (2009) aplicaron el tratamiento cognitivo conductual a una niña con estrés postraumático producto de una violación, los resultados indican una disminución en la sintomatología, llegando a niveles mínimos a partir de la novena semana, mismos resultados que se mantuvieron en el seguimiento realizados en semanas, meses y dos años después del término del tratamiento.

En otra investigación realizada por Pujadas y Pérez (2013) aplicaron el tratamiento cognitivo-conductual en un caso de estrés postraumático a causa de la muerte de un amigo. Los resultados arrojaron que el programa de tratamiento fue efectivo en su totalidad, pues lograron la total extinción de la conducta problema.

Así mismo, hay algunos tratamientos pertenecientes al modelo cognitivo conductual que se han utilizado para el trastorno de estrés postraumático.

Meichenbuan (1987) menciona el entrenamiento en inoculación al estrés como un tratamiento eficaz, pues resulta útil para los problemas subsecuentes a la exposición de sucesos estresantes como lo son las violaciones, desastres naturales, divorcios, etc. En cambio, para Foa, et al., (2002) la exposición prolongada es una estrategia terapéuticamente efectiva para disminuir los síntomas del trastorno por estrés postraumático que permite acceder a los recuerdos traumáticos a pesar del malestar temporal que presupone para pacientes con este tipo de trastornos y que en algunos casos lleva a abandonar el tratamiento.

En un estudio realizado por García (2005) a 42 miembros de fuerzas armadas se evaluó la sintomatología del estrés postraumático en diferentes momentos: antes, durante y después de aplicar un tratamiento cognitivo-conductual de exposición e inoculación al estrés. Los resultados reflejaron que las diferencias y severidad de dicho trastorno disminuyeron significativamente a partir de la segunda semana de intervención.

Gómez, et al., (2018) realizaron una intervención cognitivo-conductual de exposición prolongada a una mujer de 53 años con TEPT a raíz de un accidente ferroviario. Se fueron alcanzando los logros establecidos en la terapia en 3 y 6 meses, y en el último seguimiento a los 12 meses, realizado vía telefónica, no presento ningún síntoma de los que refirió al inicio de la terapia. Dentro de las limitaciones de este estudio, se encuentra que la paciente decidió no continuar con el tratamiento justo cuando se le planteó la exposición al recuerdo del accidente, lo que consideraron una respuesta de evitación de la propia exposición.

Por otra parte, en una investigación realizada por Guerra y Barrera (2017) implementaron el tratamiento cognitivoconductual centrado en el trauma comprobando la efectividad que tiene en adolescentes de sexo femenino de entre 12 y 17 años. Los resultados arrojaron que en 13 de las 14 participantes (92.86\%) la intervención se asocio a una disminución de la sintomatología del TEPT. Es una terapia que se aplica mayormente en niños, adolescentes y sus padres y dentro de sus limitaciones se trata de un modelo flexible que tiene que ser adaptado a las necesidades de pacientes individuales.

Con base a los estudios realizados sobre los modelos que más eficacia tienen para disminuir el TETP, el tratamiento elegido fue la terapia de inoculación al estrés empelando otras técnicas cognitivas conductuales.

Elegimos esta técnica debido a que como menciona Meichenbaun (1987) es una técnica que combina muchos elementos de enseñanza, reestructuración cognitiva, ensayos conductuales e imaginarios, entrenamiento en relajación, etc. Está creado para generar y desarrollar habilidades de afrontamiento, y no solo para resolver problemas concretos inmediatos, sino para también aplicarlo a dificultades futuras. Es por eso que decidimos aplicar este tratamiento, pues la exposición prolongada puede llevar a las pacientes a abandonar el tratamiento a 
causa de que la persona no pueda tolerar el malestar psicológico que éste implica (Foa, et al., 2002). El tratamiento centrado en el trauma tampoco sería conveniente para esta investigación debido a que de acuerdo a Guerra y Barrera (2017 ha resultado útil en niños, adolescentes y sus padres, y en este caso se realizará la intervención a mujeres de 25 a 40 años.

Se utilizara la técnica de inoculación al estrés para que las víctimas de violencia sexual puedan manejar situaciones problemáticas que les generan estrés a causa de la violencia sexual que ha sido generada con anterioridad, de esta forma podrán reaccionar de manera distinta ante situaciones futuras y no solo instantáneas que activen los síntomas de TEPT (exposición a sucesos que le recuerden al trauma, aromas, personas, lugares), así como la realización de pensamientos positivos que hagan que puedan mantenerse ante situaciones estresantes. La inoculación al estrés les enseñara a afrontar los episodios de estrés que viven a causa de la violación y como consecuencia los síntomas de TEPT. En la Tabla 1 se muestra el plan de tratamiento, demostrando los objetivos con relación a cada síntoma del TETP y las técnicas que se empelaron.

\begin{tabular}{|c|c|}
\hline Objetivo & Técnicas \\
\hline $\begin{array}{l}\text { - Promover una comprensión adecuada de } \\
\text { la experiencia traumática } \\
\text { - Promover la adaptación a la enfermedad }\end{array}$ & Psicoeducación \\
\hline $\begin{array}{l}\text { - Aumentar los niveles de afrontamiento a } \\
\text { los estímulos aversivos (personas, lugares, } \\
\text { conversaciones, actividades, objetos o } \\
\text { situaciones que despiertan recuerdos, } \\
\text { pensamientos o sentimientos angustiosos } \\
\text { relacionados al suceso traumático). } \\
\text { - Disminuir la intensidad de las reacciones } \\
\text { fisiológicas que simbolizan un aspecto del } \\
\text { suceso traumático a través del } \\
\text { mantenimiento del control de su cuerpo } \\
\text { - Disminuir el estado de alerta y reactividad } \\
\text { asociado al evento traumático. }\end{array}$ & $\begin{array}{l}\text { Exposición en } \\
\text { imaginación } \\
\text { Técnicas de } \\
\text { relajación }\end{array}$ \\
\hline $\begin{array}{l}\text { Disminuir el pensamiento catastrófico que } \\
\text { tienen respecto a si mismas, los demás o el } \\
\text { mundo ("Estoy mal", "No puedo confiar en } \\
\text { nadie", "El mundo es muy peligroso", etc.), así } \\
\text { como la percepción distorsionada que hace } \\
\text { que se culpabilicen a sí mismas o a los demás }\end{array}$ & $\begin{array}{l}\text { Restructuración } \\
\text { cognitiva }\end{array}$ \\
\hline $\begin{array}{l}\text { - Reducir el impacto emocional y estado } \\
\text { afectivo negativo (miedo, terror, enfado, } \\
\text { vergüenza) generando emociones } \\
\text { positivas (como felicidad y satisfacción) }\end{array}$ & $\begin{array}{l}\text { Reestructuració } \\
\mathrm{n} \text { cognitiva } \\
\text { Entrenamiento } \\
\text { en habilidades } \\
\text { sociales }\end{array}$ \\
\hline $\begin{array}{l}\text { - Aumentar el interés que tenía por realizar } \\
\text { actividades significativas } \\
\text { - Mejorar sus relaciones interpersonales } \\
\text { - Mejorar su calidad de vida }\end{array}$ & $\begin{array}{l}\text { Entrenamiento } \\
\text { en habilidades } \\
\text { sociales }\end{array}$ \\
\hline
\end{tabular}

\section{Implementación del tratamiento}

El entrenamiento en inoculación del estrés, es un tratamiento cognitivo-conductual orientado a proporcionarle habilidades de afrontamiento a las personas como una forma de prevenir reacciones traumáticas causadas por el estrés, se ha aplicado como estrategia terapéutica para intervenir con personas que ya sufren trastornos relacionados con el estrés erradicando o disminuyendo la sintomatología. Por lo que, su propósito es enseñarles a las pacientes habilidades que le permitan capacitarse con éxito a los problemas que le surjan en la vida diaria (Benedito y Botella, 1991; Labrado, 2008).

Se incluyen tres fases en dicho tratamiento planteadas por Meichenbaun (1987): fase de conceptualización o educativa, fase de adquisición y ensayo de habilidades y, por último, fase de aplicación y consolidación de estás.

\section{Conceptualización}

En esta fase se expone al paciente un modelo explicativo de la respuesta al estrés; en dicho modelo se deberán explicar los componentes cognitivos (lo que piensa), fisiológicos (lo que siente) y motores (lo que hace), con el objetivo de que el sujeto cambie sus cogniciones y valore e interprete la reacción al estrés de una manera más realista y adaptativa (Benedito y Botella, 1991)

En este caso se le enseñara y explicara a las pacientes cómo esas distorsiones cogniciones que tienen ("Estoy mal", "No puedo confiar en nadie", "El mundo es muy peligroso", "Fue mi culpa" "Todo fue su culpa") están afectando de manera directa en su comportamiento y en sus emociones (miedo, terror, enfado, vergüenza, conductas evitativas) y que están jugando un papel importante en el mantenimiento del problema (en sus relaciones interpersonales, vida laboral, actividades significativas que hacían antes, etc.).

\section{Adquisición y ensayo de habilidades}

En la segunda fase se comienza a entrenar aquellas habilidades que el paciente posee un menor dominio o de las que carece por completo, por lo que se le proporcionan una serie de técnicas para entrenar dichas habilidades (Benedito y Botella, 1991; Labrado, 2008). En este sentido se aplicarán las técnicas de relajación, exposición en imaginación, restructuración cognitiva y entrenamiento en habilidades sociales.

Las técnicas de relajación son un procedimiento de aprendizaje gradual que consisten en la reducción de la activación, ayudan a una persona a reducir su estado de tensión físico y/o mental facilitando la recuperación de la calma, un estado de tranquilidad y equilibrio mental (Ruiz, et al., 2012). En este caso se usará el entramiento en relajación progresiva desarrollado por Edmund Jacobson (1939) que consiste en tensar y destensar una serie de músculos empezando, en este caso, de la parte inferior a 
la superior (desde los pies hasta llegar finalmente a la cabeza) con el objetivo de que las participantes vayan percibiendo las sensaciones corporales y así inducirlas a un estado de relajación profunda. El objetivo de esto es disminuir las reacciones fisiológicas que son causadas por la simbolización del suceso traumático permitiéndoles que por si mismas puedan controlar su cuerpo y regresar a un estado de tranquilidad, disminuyendo así el estado de alerta y reactividad.

La exposición en imaginación hace referencia a enfrentar las situaciones temidas no de forma real, sino a través de las representaciones mentales (imaginación), visuales (fotografías, imágenes, películas, etc.), auditivos (grabaciones de sonidos). Su objetivo es que a través de la imaginación los sujetos puedan producir la habituación al estimulo temido, la extinción de la respuesta de miedo y así poder establecer un nuevo aprendizaje en relación al estímulo temido, facilitando así la desaparición de la conducta de evitación (Ruiz, et al., 2012). Se pretende alcanzar que las participantes puedan afrontar las conductas evitativas, a través de representaciones mentales (específicamente reproduciendo la escena del trauma con todo el detalle posible) con el objetivo de que puedan enfrentarse a las situaciones que le causen algún recuerdo del suceso traumático, ya sea algún lugar, una persona, un objeto, una conversación, etc., que lo simbolice.

La restructuración cognitiva consiste en que el paciente, con la ayuda inicial del terapeuta, identifique y cuestione sus pensamientos desadaptativos, de manera que pueda sustituirlos por otros más adaptativos y se reduzca o elimine así la alteración emocional y/o conductual causada por dichos pensamientos (Beck, 2000). Esta técnica cognitivo- conductual va a ayudar a las participantes a sustituir aquellos pensamientos disfuncionales por otros que sean más adaptativos. En este caso se sustituirán los pensamientos de "Estoy mal", "No puedo confiar en nadie", "El mundo es muy peligroso", etc. así como la percepción distorsionada que hace que se culpabilicen a sí mismas o a los demás por otros positivos como que ellas son muy afortunadas de seguir con vida, de tener a su familia, un trabajo, redes de apoyo, etc.

Por último, el entrenamiento de habilidades sociales es una técnica dirigida a enseñar estrategias y habilidades interpersonales a los individuos, está constituida por 4 tipos de habilidades sociales; asertivas, de comunicación, afectivas y cognitivas (Caballo, 1993; Labrado, 2008).

Los objetivos de esta técnica son mejorar las relaciones interpersonales, lograr que las personas adquieran habilidades de una manera socialmente aceptada y mejorar la calidad de vida (Caballo, 1993). En este caso se pretende que las pacientes puedan aumentar el interés que tenían por realizar actividades significativas, así como mejorar sus relaciones interpersonales, su calidad de vida y generar emociones más positivas, por lo que se mejoraran las habilidades asertivas, afectivas y cognitivas.

\section{Aplicación y consolidación}

En esta última fase, se prueban y practican las habilidades de entrenamiento utilizándolas realmente bajo distintas condiciones de estrés y llevándolo a la aplicación en la vida cotidiana (Benedito y Botella, 1991). En este caso se busca que las pacientes puedan desarrollar nuevas formas de reaccionar, aprendiendo a relajar y controlar por sí solas su cuerpo, sustituyendo los antiguos pensamientos desadaptativos por otros más positivos y sobre todo útiles para afrontar de manera más satisfactoria las situaciones que se les presentan relacionados al suceso traumático, una vez que se termine dicha intervención.

\section{Método}

\section{Participantes y procedimiento}

En el presente estudio participaron un total de 10 mujeres pertenecientes al municipio de Tula de Allende, Hgo.

Se desarrollo un muestreo por bola de nieve, mediante el cual, se identificaron a 3 mujeres víctimas de violación a las cuales contactamos y quienes posteriormente nos refirieron 7 personas más.

Más adelante, nos contactamos con cada una de ellas a través de llamadas para explicarles el propósito de la intervención e invitándolas a participar en ella.

La recogida de datos en esta investigación se realizó en dos fases. En la primera fase se aplicó el instrumento EGS-R para recoger información sobre el nivel de gravedad de estrés postraumático que presentan las participantes. La segunda fase se realizó después de efectuar la intervención cognitivo-conductual, aplicando nuevamente el instrumento EGS-R con el fin de apreciar si la sintomatología del estrés postraumático disminuyo o no causó ningún efecto en las participantes. Por lo tanto, la presente investigación es de tipo longitudinal, debido a que se estudió y evaluó a las mismas personas durante un periodo prolongado de tiempo para ver si hubo un cambio en la sintomatología del TETP.

\section{Variables}

La variable estrés postraumático, es lo que se investigó y midió por medio del EGS-R, es definida como una respuesta sintomatológica que una persona desarrolla en caso de haber presenciado un suceso o evento traumático que ponga en riesgo su vida o la de otra persona. La variable modelo de intervención, es la que generó un cambio para disminuir el TETP en las mujeres víctimas de una violación, se refiere a la aplicación de técnicas psicológicas por parte de un profesional, en este caso de pasantes de psicología, cuyo propósito es poder ayudar a 
las personas que lo requieran, en concreto, ayudar a las personas a afrontar sus problemas, así como disminuirlos. Instrumentos

Se utilizó el instrumento Escala de gravedad de síntomas revisada del trastorno de estrés postraumático según el DSM-V (EGS-R) (Echeburúa, 2016). El instrumento está conformado por una entrevista estructurada compuesta por 21 ítems con un formato de puntuación de escala tipo Likert con una frecuencia de 0 a 3 según la frecuencia 0 intensidad de los síntomas. Se encuentra dividida por secciones; la primera hace referencia a los síntomas de reexperimentación con un rango de 0 a 15 puntos, la segunda que se refiere a los síntomas de evitación de manera cognitiva y conductual con una rango de 0 a 9 puntos, 7 que pertenecen a alteraciones cognitivas y estados de ánimo negativos con un rango de 0 a 21 puntos, y 6 que pertenecen a los síntomas de aumento de la activación y reactividad psicofisiológica que tienen un rango de 0 a 18 puntos; de esta manera se podrá identificar si los síntomas son presentes, si la respuesta oscila entre una puntuación de 3 significará que el síntoma está presente.

\section{Resultados}

\section{Análisis descriptivo}

A continuación, se presenta por medio de gráficas la gravedad de la sintomatología del TEPT tras la primera aplicación del instrumento EGS-R a la muestra de 10 mujeres.

En la figura 1 se muestran los síntomas de reexperimentación.

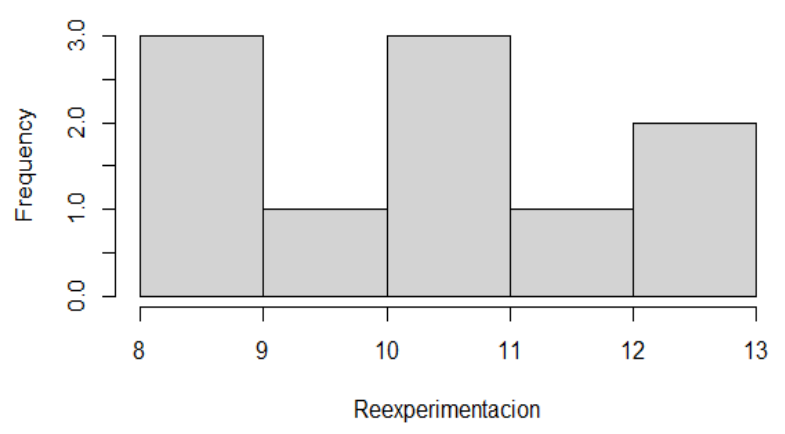

Figura 1. Síntomas de reexperimentación

De un rango de 0 a 15 puntos de los síntomas de reexperimentación, el mayor puntaje obtenido fue de 1213 puntos por 2 personas, seguido de una mujer con una puntuación entre 11 y 12 , y tres mujeres con una puntuación de 10 y 11 puntos. El menor puntaje obtenido fue de 8 y 9 puntos alcanzados por tres mujeres, y una puntuación de 9-10 por únicamente una mujer.
En la figura 2 se muestran los síntomas de evitación conductual/cognitivo.

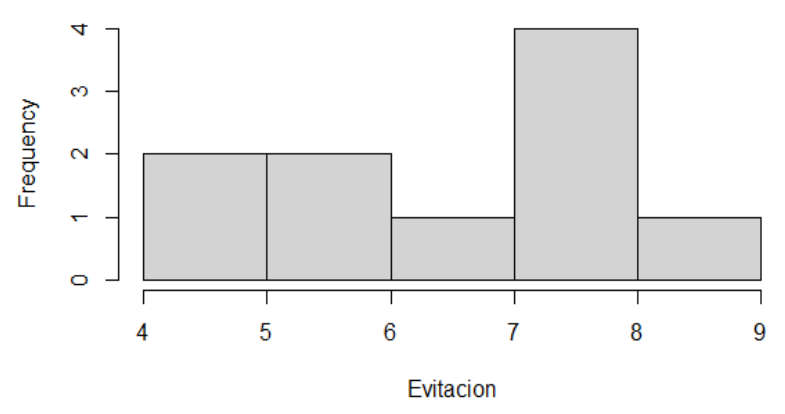

Figura 2. Síntomas de evitación conductual/cognitiva

De un rango de 0 a 9 puntos, dos mujeres obtuvieron una puntuación de 4 y 5 puntos y dos mujeres una de 5 y 6 puntos. Únicamente una mujer obtuvo una puntuación de 6-7 puntos y otra mujer una de 8-9 puntos. La frecuencia más alta se encuentra entre los 7 y 8 puntos obtenidos por 4 mujeres.

En la figura 3 se muestran los alteraciones cognitivas y estado de ánimo negativo

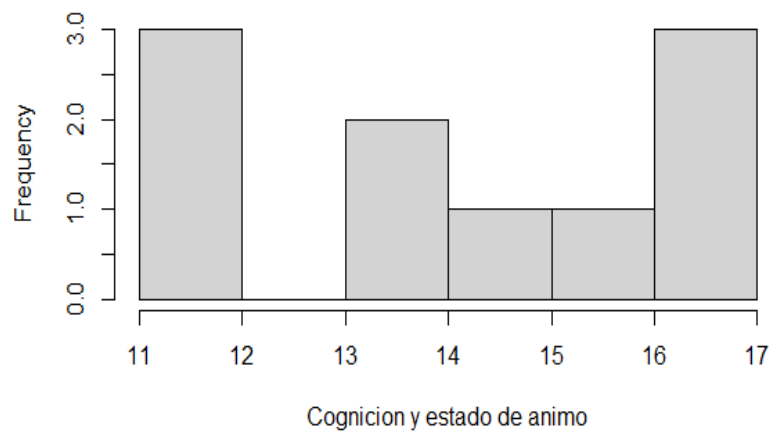
Figura 3. Alteraciones cognitivas y estado de ánimo
negativo

De un rango del 0 a 21 puntos tres mujeres obtuvieron una puntuación de 11 y 12 puntos, dos mujeres de 13-14 puntos, una mujer con puntuación de 14 y 15 puntos, y otra entre 15-16 puntos. La puntuación más alta fue de 1617 puntos obtenidos por tres de ellas en esta sección. En la figura 4 se observan los resultados de la sección aumento de la activación y reactividad psicofisiológica. 


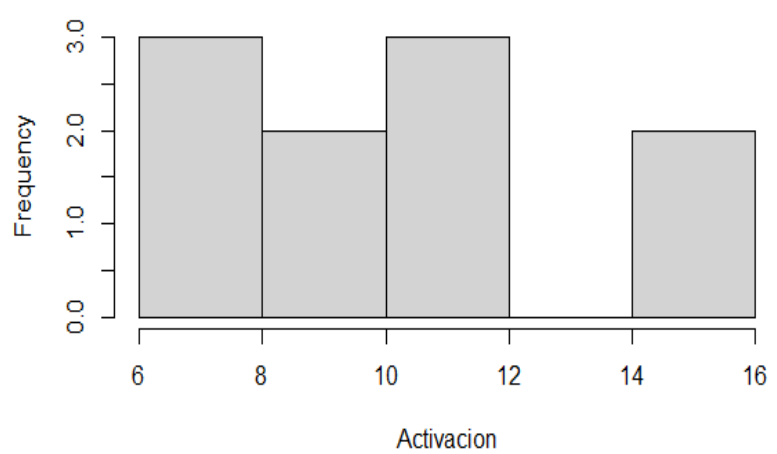

Figura 4. Aumento de la activación y reactividad psicofisiológica

De un rango de 0 a 18 puntos, tres mujeres arrojaron una puntuación de 6-8 puntos, dos mujeres obtuvieron entre 8 y 10 puntos, tres alcanzaron un puntaje entre 10 y 12 y dos una puntuación de 14-16 en la sección.

En la figura 5 se muestran los resultados con respecto a los síntomas disociativos.

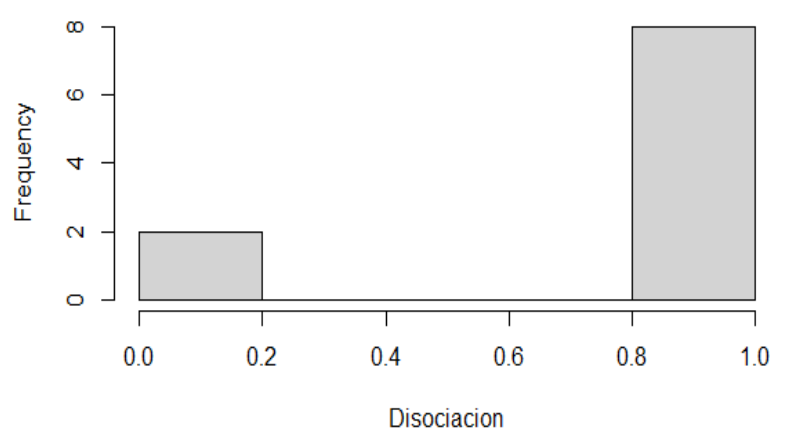

Figura 5. Síntomas disociativos

De un rango de 0 a 1 punto en esta sección, donde 1 representa si y 0 no, fueron únicamente 8 mujeres las que presentaron síntomas disociativos, mientras que las dos restantes no presentan síntomas disociativos.

En la figura 6 se observan los resultados de la disfuncionalidad en la vida cotidiana relacionada con el suceso traumático.

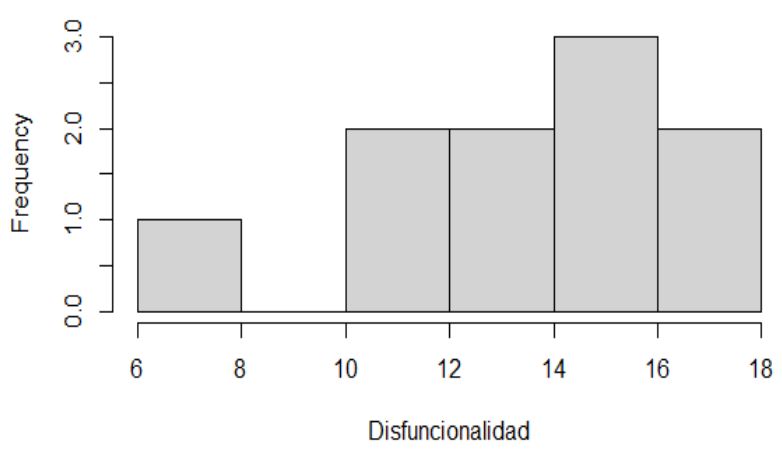

Figura 6. Disfuncionalidad en la vida cotidiana

De un rango de 0 a 18 puntos, la puntuación más alta se encuentra entre los 14 y 16 puntos siendo tres mujeres la que la obtuvieron, seguida de dos que obtuvieron entre 16 y 18 . Cuatro mujeres obtuvieron de 10 a 14 puntos y una de 6 a 8.

De la primera aplicación de la escala de gravedad de síntomas del trastorno de estrés postraumático- revisada (EGS-R), se concluye que la muestra de 10 mujeres cumple con los criterios del diagnóstico de estrés postraumático, sumado a ello 8 de ellas también presentaron síntomas disociativos y todas presentan disfuncionalidad en su vida cotidiana a causa del suceso traumático, por lo tanto, pasaron a la fase de tratamiento.

\section{Análisis inferencial}

En la segunda fase, se implementó el tratamiento y al concluir se aplicó nuevamente el EGS-R. Con la finalidad de conocer si había diferencias significativas en cuanto a la sintomatología antes y después de dicho tratamiento, se utilizó la prueba $t$ de Student de comparación de medias para muestras independientes. Los datos se analizaron mediante el Programa Estadístico para Ciencias Sociales (SPSS $\circledast$ ).

Como se observa en tabla 2, al aplicar la prueba antes y después, se obtuvo el resultado de $t=18.239, p<.0001$ en los síntomas de reexperimentación, $t=13.679, p<$ .0001 en evitación conductual/cognitiva, $t=18.872, p<$ .0001 en alteraciones cognitivas y estado de ánimo negativo, $t=11.658, p<.0001$ en aumento de la activación $y$ reactividad psicofisiológica, $t=6.000, p<.0001$ en síntomas disociativos y $\mathrm{t}=13.451, \mathrm{p}<.0001$ en disfuncionalidad en la vida cotidiana. 


\begin{tabular}{|l|c|c|c|c|}
\hline \multicolumn{1}{|c|}{ Síntoma } & M pre & M post & $\mathbf{t}$ & $\mathbf{P}$ \\
\hline Reexperimentación & 10.60 & 5.90 & 18.239 & .000 \\
\hline $\begin{array}{l}\text { Evitación } \\
\text { conductual/cognitiva }\end{array}$ & 6.90 & 2.80 & 13.679 & .000 \\
\hline $\begin{array}{l}\text { Alteraciones } \\
\text { cognitivas y estado } \\
\text { de ánimo negativo }\end{array}$ & 14.40 & 5.50 & 18.872 & .000 \\
\hline $\begin{array}{l}\text { Aumento de la } \\
\text { activación y } \\
\text { reactividad } \\
\text { psicofisiológica }\end{array}$ & 10.60 & 3.0 & 11.658 & .000 \\
\hline Disociación & .80 & .40 & 6.000 & .000 \\
\hline $\begin{array}{l}\text { Disfuncionalidad en } \\
\text { la vida cotidiana }\end{array}$ & 14.10 & 3.50 & 13.451 & .000 \\
\hline
\end{tabular}

técnicas de relajación y entrenamiento en habilidades sociales en mujeres víctimas de una violación fue efectiva en la reducción de síntomas.

\section{Referencias}

American Psychiatric Association. (2014). Manual diagnóstico y estadístico de los trastornos mentales ( $5^{\mathbf{a}}$ ed.). Madrid, España: Médica Panamericana.

Baguena, M. J. (2001). Tratamientos psicológicos eficaces para el estrés postraumático. Psicothema, 13 (3), 479- 492.

Benetido, C. y Botella, C. (1991). Entrenamiento en inoculación de estrés en un caso de asma infantil. Análisis y modificación de conducta, 17 (55), 840-849.

Beck, J. (2000). Terapia cognitiva: Conceptos básicos y profundización. España: Edisa.

Con base a lo antes plateado, se confirma que existen diferencias significativas en la sintomatología del TEPT antes y después de aplicar el tratamiento de inoculación al estrés, siendo menores las medias después de aplicar el tratamiento y mayores antes de aplicarlo.

\section{Discusión}

Los resultados de este trabajo coinciden con las investigaciones de Cáceres, et al., (2011), Guerra y Plaza (2009) y Pujadas y Pérez (2013), respecto a que, en efecto el modelo de intervención cognitivo conductual ha resultado útil y eficaz para disminuir el cuadro clínico del TEPT. Así mismo coinciden con los estudios realizados por Meichenbuan (1987) y García (2002) confirmando que el entrenamiento de inoculación al estrés es eficaz para la disminución de síntomas del TEPT, específicamente para el problema subsecuente de un suceso estresante como lo es una violación sexual.

\section{Conclusión}

De acuerdo con el análisis de los datos recogidos en las evaluaciones de las 10 mujeres víctimas de violación sexual, que participaron en el tratamiento de inoculación al estrés, se evidenciaron diferencias significativas en la sintomatología del TEPT antes y después de aplicar la EGS-R de Echeburúa (2016).

Los resultados indicaron que se alcanzaron todos los objetivos planteados en el tratamiento, así mismo, se cumplió la hipótesis de la investigación.

Los resultados arrojan que las mujeres que cumplían con el cuadro clínico de TEPT disminuyeron la frecuencia e intensidad de su sintomatología, y su funcionabilidad en la vida cotidiana mejoró: volvieron a retomar actividades que habían dejado de hacer, la relación con su pareja, familia, su vida laboral y social aumentaron. Para concluir, se puede afirmar que la intervención cognitiva conductual de inoculación al estrés empleando las técnicas de restructuración cognitiva, exposición en imaginación,

Caballo, V. (1993). Manual de evaluación y entrenamiento de las habilidades sociales. España: Siglo XXI de España editores.

Cáceres, E., Labrador, F., Ardilla, P. y Parada, D. (2011). Tratamiento cognitivo conductual centrado en el trauma en mujeres víctimas de violencia de pareja. Psychologia Avances de la disciplina, 5 (2), 13-31. http://www.scielo.org.co/pdf/psych/v5n2/v5n2a02.pdf

Echeburúa, E., Amor, P., Sarasua, B., Zubizarreta, I., Holgado, F. y Muñoz, M. (2016). Escala de Gravedad de Síntomas Revisada (EGS-R) del Trastorno de estrés postraumático según el DSM-5: propiedades psicométricas. Terapia psicológica, 34 (2), 111.128.

Echeburúa, E. y Amor, P. (2019). Memoria traumática: estrategias de afrontamiento adaptativas e inadaptativas. Terapia Psicológica, 37 (1). Recuperado

https://scielo.conicyt.cl/scielo.php?script=sci_arttext\&pid=S071848082019000100071

Foa, E., Zoellner, L., Feeny, N., Hembree, E. \& AlvarezConrad, J. (2002). Does imaginal exposure exacerbate PTSD symptoms?. Journal of Consulting and Clinical Psychology, 70, 1022-1028.

Gabalda, I. (2009). Manual teórico-práctico de Psicoterapias Cognitivas (2a edición). España: Desclée de Brouwer.

García, C. (2005). Efectividad de una intervención cognitivo-conductual para el trastorno de estrés postraumático en excombatientes colombianos. Universitas Psychologica, 4 (2), 205-219.

Guerra, C. y Barrera, P. (2017). Psicoterapia con víctimas de abuso sexual inspirada en la terapia cognitivo-conductual centrada en el trauma. Revista de psicología, 26 (2), p. 1-13. Recuperado de https://scielo.conicyt.cl/pdf/revpsicol/v26n2/0719-0581-revpsicol-2602-00016.pdf

Guerra, C. y Plaza, H. (2009). Tratamiento Cognitivo-Conductual del Estrés Postraumático en un caso de violación infantil. Revista de Psicología, 18 (1), 103-125. file:///C:/Users/52477/Downloads/17130-1-50141-1$10-20111106 \% 20(1) . p d f$

González, C. y Javato, L. (2015). Eficacia del tratamiento cognitivoconductual para un caso de bulimia. Revista de Casos Clínicos en Salud Mental, 1, 19-41.

Gómez, M., Chaparro, G., Francisco, C. y Crespo, M. (2018). Tratamiento cognitivo conductual de un caso de estrés postraumático por accidente 
ferroviario: ¿Éxito terapéutico o evitación?. Clínica y salud, 29 (2). Recuperado http://scielo.isciii.es/scielo.php?script=sci_arttext\&pid=S113052742018000200101

Herman. J. (2015). Trauma and Recovery: The Afermath of Violence-From Domestic Abuse to Political Terror. Nueva York: Basic Books.

Instituto Nacional de Estadística y Geografía (2016). Estadísticas a propósito del día internacional de la eliminación de la violencia contra la mujer. Recuperado de https://www.inegi.org.mx/contenidos/saladeprensa/aproposito/2020/Vi olencia2020_Nal.pdf?fbclid=IwAR2hD5mR1ZesgmWv1zCf6Ww1ms _ohgA2ubrA9whsw1jFfMcI-iHKuUyOHbA

Jackson, H. J., McGorry, P. D., Killackey, E., Bendall, S., Allott, K., Dudgeon, P., et al. (2008). Acute phase and 1-year follow-up results of a randomized controlled trial of CBT versus befriending for firstepisode psychosis: The ACE project. Psychological Medicine: A Journal of Research in Psychiatry and the Allied Sciences, 38 (5), 725735 .

Kardiner, A. (2012). The Traumatic Neuroses of War. Nueva York: Martino Fine Books.

Lozano, J.F., Gómez, M., Avilés, C. y Sepúlveda, A. (2018). Sintomatología del trastorno de estrés postraumático en una muestra de mujeres víctimas de violencia sexual. Curanderos de medica forense, 23 (3). Recuperado de http://scielo.isciii.es/scielo.php?script=sci_abstract\&pid=S1135$76062017000200082 \& \operatorname{lng}=$ es\&nrm=iso

Labrador, F. (2008). Técnicas de modificación de conducta. España: Pirámide.

Meichembaun, D. (1987). Manual de Inoculación de Estrés. España: Martínez Roca.

Medina, E., Borges, G., Lara, C., Ramos, L., Zambrano, J. y Fleiz, C. (2005). Prevalencia de sucesos violentos y de trastorno por estrés postraumático en la población mexicana. Salud pública de México, 47 (1). Recuperado de http://www.scielo.org.mx/scielo.php?script=sci_arttext\&pid=S003636342005000100004

Muruaga, S. (11 de Junio de 2018). Efectos de la violencia sexual en las mujeres. Mujeres para la salud. Recuperado de https://www.mujeresparalasalud.org/efectos-de-la-violencia-sexual-enlas-mujeres/

Palacios L. y Heinze, G. (2002).Trastorno por estrés postraumático: una revisión del tema (primera parte). Salud mental, 25 (3), 19-26. Recuperado de https://www.medigraphic.com/pdfs/salmen/sam2002/sam023c.pdf

Puerta, J. V. y Padilla, D. E. (2011). Terapia cognitiva - conductual (TCC) como tratamiento para la depresión: una revisión del estado del arte. $\begin{array}{llll}\text { Duazary, } & 8 & \text { (2), 251-257. }\end{array}$ https://www.redalyc.org/pdf/5121/512156315016.pdf

Padilla, C. (2014). Tratamiento cognitivo-conductual de un adolescente con trastorno de ansiedad generalizada. Revista de Psicología Clínica con Niños y Adolescentes, 1 (2), 157-163.

Pujadas, M. y Pérez, J. (2013). Tratamiento de un caso por estrés postraumático. Revista de casos clínicos en Salud Mental, 1, 37-58.

Ruiz, M., Díaz, M. y Villalobos, A. (2012). Manual de técnicas de intervención Cognitivo-Conductuales. México: Desclée De Brouwer.
Ramos, L., Saltijeral, M., Romero, M., Caballero, M. y Martínez, N. (2001) Violencia sexual y problemas asociados en una muestra de usuarias de un centro de salud. Salud Pública de México, 43(3), 182-191. Recuperado de http://www.scielo.org.mx/scielo.php?script=sci_arttext\&pid=S0036$36342001000300002 \& \operatorname{lng}=e s \&$ tlng $=e s$

Rozo, J. A. (2019). Psicología Contemporánea: Tendencias y aplicaciones en la Psicología del siglo XXI. Bogotá: Biblomedia Editores.

Travé, J. y Pousa, E. (2012). Eficacia de la Terapia Cognitivo-Conductual en pacientes con psicosis de inicio reciente: una revisión. Papeles del psicólogo, $33 \quad$ (1), 48-59. https://www.redalyc.org/pdf/778/77823404006.pdf

World Health Organization. (2013). Estimaciones mundiales y regionales de la violencia contra la mujer: prevalencia y efectos de la violencia conyugal y de la violencia sexual no conyugal en la salud. Recuperado de

https://apps.who.int/iris/bitstream/handle/10665/85243/WHO_RHR_H RP_13.06_spa.pdf?sequence=1

World Health Organization. (2017). Violencia contra la mujer. Recuperado de https://www.who.int/es/news-room/fact-sheets/detail/violenceagainst-women [1] Dilla T, Valladares A, Lizán L, Sacristán JA. Adherencia y persistencia terapéutica: causas, consecuencias y estrategias de mejora. Aten. Primaria 2009; 41(6): 342-48. 